\title{
Hemispheric asymmetries in global/local processing are modulated by perceptual salience
}

\author{
G. R. Fink ${ }^{\mathrm{a}, \mathrm{b} *}$, J. C. Marshall ${ }^{\mathrm{c}}$, P. W. Halligan ${ }^{\mathrm{c}, \mathrm{d}}$, R. J. Dolan ${ }^{\mathrm{a}, \mathrm{e}}$ \\ ${ }^{a}$ Wellcome Department of Cognitive Neurology, Institute of Neurology, London, UK \\ ${ }^{\mathrm{b}}$ Neurologische Klinik, Heinrich-Heine-Universität, Düsseldorf, Germany \\ ${ }^{\mathrm{c}}$ Neuropsychology Unit, The Radcliffe Infirmary, Oxford, UK \\ ${ }^{\mathrm{d}}$ Department of Experimental Psychology, Oxford, UK; and ${ }^{\mathrm{e}}$ Royal Free Hospital School of Medicine, London, UK
}

(Received 4 December 1997; accepted 5 May 1998)

\begin{abstract}
It has been claimed that a left hemisphere bias toward local and right hemisphere bias toward global visual processing can be explained in terms of specialization for relatively high and low spatial frequencies, respectively. Using non-representational figures, we tested this hypothesis in experiment 1 using positron emission tomography (PET) measures of cerebral activity in 10 normal volunteers. In each block of trials subjects viewed either a relatively high or a relatively low spatial frequency grating. The orientation (vertical or horizontal) of the grating changed from trial to trial. In a directed attention task, subjects reported the orientation of either the whole stimulus (globally directed attention) or the orientation of the component parts thereof (locally directed attention). A significant interaction between hierarchical processing level (global or local) and stimulus level (relatively high or relatively low spatial frequency within the absolute low spatial frequency range) was found. Globally directed attention led to significantly increased cerebral activity in the right hemisphere when relatively high spatial frequency stimuli were used but not when relatively low spatial frequency stimuli were used. Likewise, locally directed attention increased cerebral activity when low but not high spatial frequency stimuli were used. On the basis of these results we suggest that perceptual salience of the global or local form modulates hemispheric processing asymmetries in early visual cortex. In experiment 2, the perceptual salience of global form in relatively high spatial frequency stimuli and of local form in relatively low spatial frequency stimuli was confirmed in a reaction time (RT) study. In combination, the results of the two experiments suggest that perceptual salience takes precedence over spatial frequency (within the range studied here) in determining the cerebral organization of global/local processing. (C) 1998 Elsevier Science Ltd. All rights reserved.
\end{abstract}

Keywords: Spatial frequency; Early visual cortex; Hemispheric interaction; Directed visual attention; Functional imaging

\section{Introduction}

Neuropsychological [32, 33] and functional imaging studies $[5,6]$ have shown a right hemisphere advantage for global processing (the perception of and attention to the whole) and a left hemisphere advantage for local processing (the perception of and attention to the focal components that make up the whole). This hemispheric specialization, however, is relative rather than absolute $[5,6,29]$ and is greatly influenced by experimental conditions [21] and subjects' attentional set [19]. Such observations suggest that perceptual and attentional

*Corresponding author. Tel.: -49-211-81-16322; fax: -49-211-8118485; e-mail: finkg@mail.rz.uni-duesseldorf.de mechanisms interact in the processing of hierarchically organized visual stimuli.

Converging evidence from lesion and functional imaging data indicates that the functional anatomy of global/local processing involves attentional control mediated via temporal-parietal areas over neural transformations occurring in early visual cortex [5, 6, 32, 33]. The most extensively worked out model of attentional control in global/local processing is that of Robertson and Lamb [32]. This four-component model incorporates a global processor located in the right posterior superior temporo-parietal cortex, a local processor associated with the homologous left hemisphere region, a bilateral parietal lobe system that controls the distribution of attentional resources to the global and local levels and posterior temporal-temporal pathways that com- 
municate between the subsystems and fine tune them. The evidence for these temporo-parietal systems has come from paradigms that require subjects to attend to both global and local levels (divided attention tasks) at the same time $[6,22]$. By contrast, tasks in which attention is directed to one level (either global or local), have found early visual areas to be implicated [6, 24].

Nonetheless, the factors that determine hemispheric specialization for such hierarchical processing remain poorly understood. One influential account proposed that the observed hemispheric differences resulted from differential sensitivity to spatial frequency characteristics [37], with a right hemisphere bias toward processing the low spatial frequency (global) components and a left hemisphere bias toward high spatial frequency (local) components of a visual scene. There is also evidence that stimulus category (letters or meaningful objects) influences processing asymmetries in early visual areas [5-7]. We have previously demonstrated a reversal of hemispheric asymmetry for local and global processing with a change in stimulus category (from letters to meaningful objects [7]). Although there were sensory differences between the two classes of stimuli used, we conjectured that the primary determinant of the reversal was a topdown effect: local processing of letters is, in part, driven from left hemisphere regions specialized for language, while local processing of meaningful objects is, in part, driven from right hemisphere regions specialized for object-recognition.

The first experiment of our current study further explores these issues by examining the effects of changes in spatial frequency on neural responses associated with tasks that required global and local processing of nonrepresentational figures. Relatively high or relatively low spatial frequency stimuli were used, with each stimulus containing one local and one global level. The sole requirement for subjects was to attend and report either the global or the local level of each stimulus. Within models that postulate an "attentional spotlight" [16] or a "zoom lens" [23] global processing implicates expansion of the "spotlight"/"zoom lens" while local processing implicates shrinking thereof. The current experiment investigates in these terms two distinct settings of the "spotlight"/"zoom lens" but not the process of changing their field of view.

We used PET to index, in normal human subjects, cerebral activity associated with the globally and locally directed attentional tasks by measuring changes in relative regional cerebral blood flow (rCBF) [31]. Based on previous investigations of visual attention, our own studies on global/local processing [5-7], other functional imaging studies $[2,4,38]$ and electrophysiological studies in animals $[26,27,40]$, we predicted that the modulatory effect of visual attention would result in changes in cerebral activity in early visual processing areas. Previous neuropsychological evidence suggests that changes in spatial frequency may interact with the effects of visual attention [37].

\section{Experiment 1: a PET study}

\subsection{Method}

\subsubsection{Subjects.}

Ten normal healthy volunteers ( 9 male, 1 female; aged 22-65 years) were recruited. All were right-handed with no history, past or current, of neurological or psychiatric illness. Informed written consent was obtained from all subjects. The study involved administration of $4.5 \mathrm{mSv}$ effective dose equivalent of radioactivity per subject. Permission to administer radioactivity was obtained from the Administration of Radioactive Substances Advisory Committee of the Department of Health, U.K. The study was approved by the local ethics committee of the National Hospital for Neurology and Neurosurgery (London, U.K.).

\subsubsection{Paradigm design}

This experiment was designed to be as similar as possible to our previous studies of letter and object hierarchical stimuli [5-7]. In those investigations we required the subjects to name the target stimuli. For this reason we have retained (in Experiment 1) a verbal, rather than a manual reaction time, response mode in this experiment. The experimental design and examples of the figures used as stimuli during the globally and locally directed attention tasks are shown in Fig. 1.

To study spatial frequency effects, both relatively high and relatively low spatial frequency stimuli were displayed. The stimuli were approximations to sine waves. Truncated (co)sine wave forms were used with the truncation set at $50 \%$. Each stimulus consisted of either 6 cycles or 3 cycles of the waveform per $16^{\circ}$ of visual angle. Although these gratings are relatively high and low spatial frequency respectively, both fall within the overall low range (less than 1 cycle/degree) of spatial frequency. These gratings were chosen for three reasons. First, their abstract nature; they are neither letters nor meaningful objects. Second, the local forms are more visible in these gratings than in pure sine wave stimuli. Third, we were concerned with how relative spatial frequency might modulate global-local processing (and not with the more general effects of absolute spatial frequencies on perceptual processing). The stimuli were displayed on a white background (rather than a gray that had the average luminance of black and white) in order to enhance the visibility of both the shape as a whole and its component parts.

In each block of trials, subjects were presented with either a relatively high or a relatively low spatial frequency grating whose orientation (vertical or horizontal) changed from trial to trial (Fig. 1). In a directed attention 


\section{directed attention task}

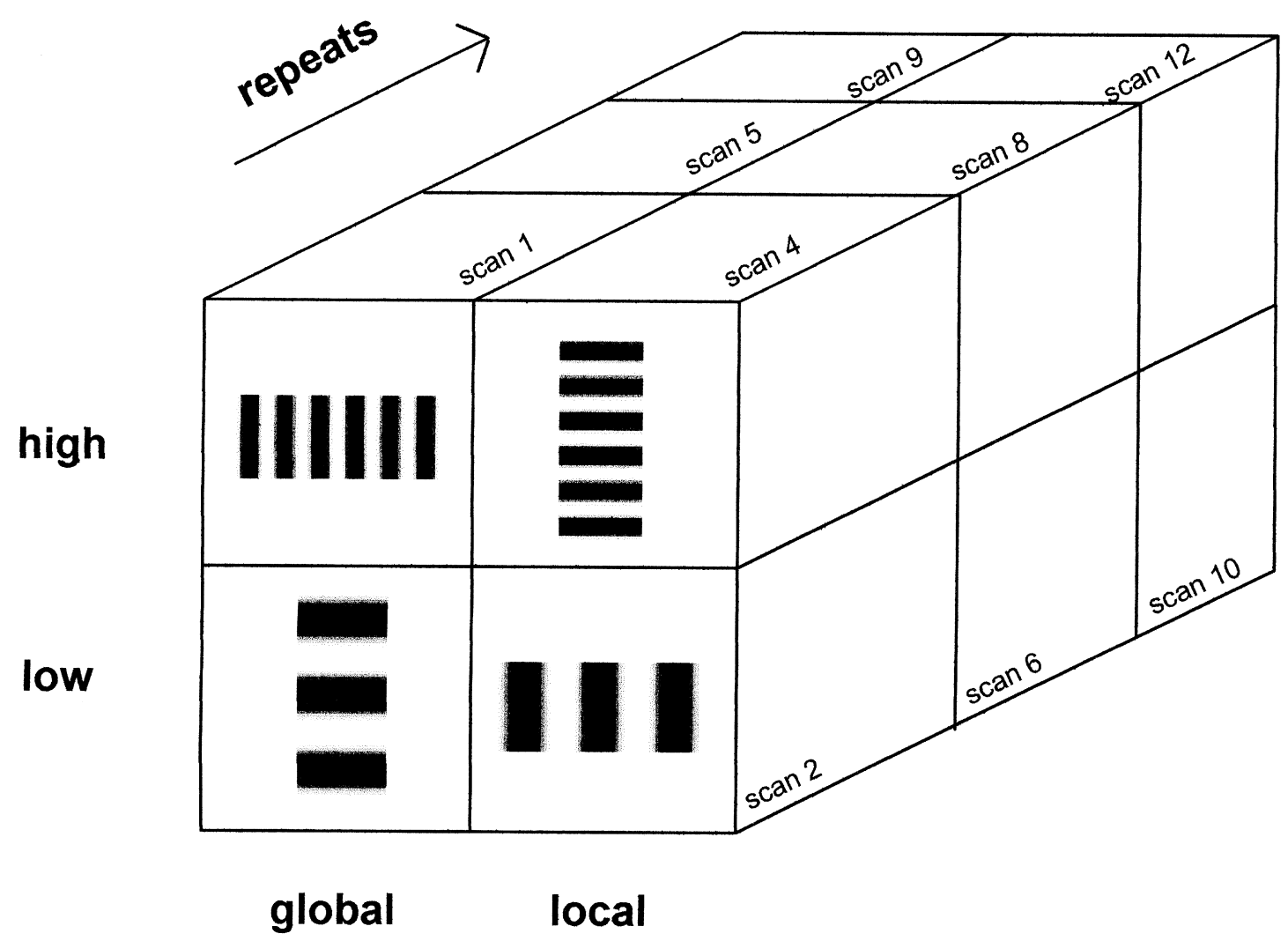

Fig. 1. The figure shows the study design and the high (top row) and low (bottom row) spatial frequency stimuli used. All stimuli were displayed either horizontally or vertically and were non-congruent for the global and local level. During the tasks subjects were required to attend to either the global or the local level and to name the orientation of that level ("horizontal", "vertical"). Scan numbers refer to the order of scans for subject 1 (of the group of 10 subjects studied). The order of scans was counter-balanced across subjects.

task, subjects had to report the orientation of either the stimulus as a whole (globally directed attention) or the orientation of the component parts (locally directed attention). All figures were non-congruent for the orientation of global and local levels. When (for example) the local parts were horizontally oriented, the global figure had a vertical orientation. In a given block of trials, a quasi-random sequence prevented any stimulus level (local or global) from appearing horizontally (or vertically) more than five times on successive trials. Subjects lay with eyes open in the PET (positron emission tomography) scanner in a quiet, darkened room. Relative regional cerebral blood flow ( $\mathrm{rCBF}$ ) measurements were taken while the stimuli were presented on a white background. The visual angle engaged was $16^{\circ}$. A stimulus appeared every $1.5 \mathrm{~s}$ and remained for $300 \mathrm{~ms}$, in the centre of a 17" video display unit at a viewing distance of $40 \mathrm{~cm}$. The task was performed in free vision to avoid introducing the further local level of a fixation dot [5-7]. Subjects were instructed to attend either to the global or the local attribute of the stimulus and to name its orientation ("horizontal" or "vertical"). Prior to PET scanning, all subjects underwent a familiarization session to ensure fluency in task performance. The experiment involved 12 sequential relative $\mathrm{rCBF}$ measurements per subject. Each sequence of stimulus presentations began $10 \mathrm{~s}$ prior to PET scanning and lasted for $120 \mathrm{~s}$. The four testing conditions were presented in a fully factorial design: (HG) high spatial frequency stimuli, globally directed attention; (HL) high frequency stimuli, locally directed attention; (LG) low frequency stimuli, globally directed attention; (LL) low frequency stimuli, locally directed attention. Each condition was repeated three times in 10 subjects. To control for time effects, the experimental conditions were counterbalanced both within and between subjects. 
Thus the experiment employed a fully crossed factorial design in which each factor had two levels (processing level: global, local; stimulus level: relatively high spatial frequency, relatively low spatial frequency; see Fig. 1). This gave a total of four different conditions and three replications for each of these conditions across the 12 scans (per subject). Therefore, by virtue of the factorial design, the main effects and interaction terms are based on 12 scans (per subject). As 10 subjects were studied each contrast was thus based on 120 scans. Simple main effects were based on six scans per subject and therefore a total of 60 scans. The factorial experimental design (Fig. 1) crossing stimulus level with processing level ensured that identical visual stimuli were presented across all relevant comparisons.

No low-level perceptual task was employed as a baseline condition. There were two reasons for this decision: First, the addition of another condition would have resulted in a loss of statistical power (due to a reduced number of repeats per condition within the maximum of 12 scans per subject). Second, our primary concern was to image the effects of change in relative spatial frequency on hemispheric effects of global/local processing, in particular in prestriate centres. In this respect we would not expect to reveal areas activated in common by global and local processing (i.e. areas activated relative to a lowlevel control).

\subsubsection{PET-scanning}

Relative $\mathrm{rCBF}$ was measured by recording the regional distribution of cerebral radioactivity following the intravenous injection of ${ }^{15} \mathrm{O}$-labelled water $\left({ }^{15} \mathrm{O}\right.$ is a positron emitter with a half-life of $2.1 \mathrm{~min}$; [25]). The PET measurements were carried out using a SIEMENS/CPS ECAT EXACT HR + (model 962) PET scanner (CTI Inc., Knoxville, TN, U.S.A.) with a total axial field of view of $155 \mathrm{~mm}$ covering the whole brain. Data were acquired in 3-dimensional mode [42] with interdetector collimating septa removed and a Neuro-Insert installed to limit the acceptance of events originating from out-offield-of-view activity (from the whole body).

For each measurement of relative $\mathrm{rCBF}, 9 \mathrm{mCi}$ of $\mathrm{H}_{2}{ }^{15} \mathrm{O}$ were given i.v. as a slow bolus over 20 s [39]. Twelve consecutive PET scans were collected, each beginning with a $30 \mathrm{~s}$ background scan before the delivery of the slow bolus. Emission data were thereafter collected sequentially over $90 \mathrm{~s}$ after tracer arrival in the brain and corrected for background. This process was repeated for each emission scan with 8 min between scans to allow for adequate decay of radioactivity. All emission scan data were corrected for the effects of radiation attenuation (by the skull, for example) by means of a transmission scan taken prior to the first relative $\mathrm{rCBF}$ measurement. The corrected data were reconstructed into 63 transverse planes (separation $2.4 \mathrm{~mm}$ ) and into $128 \times 128$ pixels (size $2.1 \mathrm{~mm}$ ) by 3 -dimension filtered back projection using a
Hann filter of cutoff frequency 0.5 cycles per pixel and applying a scatter correction. The resolution of the resulting PET images was $6 \mathrm{~mm}$ (at full width half maximum).

\subsubsection{Magnetic resonance imaging}

In separate sessions, a magnetic resonance (MR) image of each subject's brain was obtained (a) to exclude the possibility of morphological/pathological abnormalities and (b) for stereotactic normalization into the standard anatomical space (see below). This imaging was performed with a 2 Tesla system (VISION, SIEMENS AG, Germany) using a 3-dimensional T1 weighted imaging technique producing 108 transaxial slices $(1 \times 1 \times 1.5 \mathrm{~mm})$ which gave high grey to white matter contrast.

\subsubsection{Image processing}

All calculations and image manipulations were performed on SPARC workstations (SUN Computers). PROMATLAB software (MATHWORKS Inc., U.S.A.) was used to calculate and display images. Statistical parametric mapping (SPM) software (SPM95; Wellcome Department of Cognitive Neurology, London, U.K.) was used for image realignment, image normalization, smoothing and to create statistical maps of significant relative rCBF changes $[8,9]$.

\subsubsection{Realignment, transformation and smoothing of PET images}

Using SPM95 software [8] all PET scans were realigned to the first emission scan to correct for head movement. A mean relative $\mathrm{rCBF}$ image was created for each subject. Each individual's MR image and PET mean image (serving as a template for the individual PET images) were then transformed into a standard stereotactic anatomical space $[8,41]$ using linear proportions and a non-linear sampling algorithm. The PET images were thereafter filtered using a low-pass Gaussian filter (resulting in an image resolution of $12 \mathrm{~mm}$ ) to reduce the variance due to individual anatomical variability and to improve signal-to-noise ratio [8]. The resulting pixel size in stereotactic space was $2 \times 2 \mathrm{~mm}$ with an interplane distance of $4 \mathrm{~mm}$. Data were thereafter expressed in terms of standard stereotactic coordinates in the $x$-, $y$ - and $z$-axes.

\subsubsection{Statistical analysis}

Following stereotactic normalization and image smoothing, statistical analysis was performed. The main effects of test conditions (spatial frequency, globally/locally directed attention), their interaction with one another and the simple effects of each condition were estimated on a pixel-by-pixel basis using SPM95 [9]. Task related differences in overall CBF, within and between subjects, were removed by treating overall activity as the covariate [9]. This manipulation removed systematic state-dependent differences in overall blood flow associated with the different conditions which can obscure task related 
regional alterations in activity. For each pixel in stereotactic space the ANCOVA generated a condition specific adjusted mean $\mathrm{rCBF}$ value (arbitrarily normalized to $50 \mathrm{ml} / 100 \mathrm{ml} / \mathrm{min}$ ) and an associated adjusted error variance [9]. This allowed the planned comparisons of the mean blood flow distributions across all sets of conditions. For each pixel, across all subjects and all scans, the mean relative $\mathrm{rCBF}$ values were calculated separately for each of the conditions. Comparisons of the means were made using the $t$ statistic and thereafter transformed into normally distributed $Z$ statistics. The resulting set of $z$-values constituted a statistical parametric map $\left(\mathrm{SPM}_{\{\}_{\}^{-}}}\right.$ map) [9]. For the contrasts of interest, the significance of these statistical parametric maps was assessed by comparing the expected and observed distribution of the $t$ statistic under the null hypothesis of no differential activation effect on $\mathrm{rCBF}$. Only activations that were within the early visual processing areas or the attention system and which were significant in the above described sense at $P<0.001$ or better are reported. Other "non-predicted" activations outside the early visual processing areas or the attentional system were observed but are only reported if they were significant at $P<0.05$ corrected for multiple comparisons according to the method described by Friston et al. [9]. The data were analysed for the two main effects (relatively high and low spatial frequency; globally and locally directed attention), their interaction and the simple main effects; these comparisons were intended to identify those cortical areas concerned with the properties in question (high and low spatial frequency; globally and locally directed attention). To assess hemispheric asymmetries in $\mathrm{rCBF}$ responses, images were flipped from left to right and hemisphere $\times$ condition interactions were identified using SPM95.

\subsubsection{Localization of activations}

The stereotactic coordinates of the pixels of local maximum significant changes in relative $\mathrm{rCBF}$ within areas of significant relative $\mathrm{rCBF}$ change associated with the different tasks were determined. The anatomical localization of these local maxima was assessed by reference to the standard stereotactic atlas of Talairach and Tournoux [41]. Additional validation of this method of localization was obtained after superimposition of the $\mathrm{SPM}_{\{z\}}$-maps on the group mean MR image calculated after each individual's MR image had been stereotactically transformed into the same standard stereotactic space [8].

\subsection{Results}

The main effects of stimulus level (relatively high vs relatively low spatial frequency and vice-versa) were initially assessed. High spatial frequency stimuli elicited (relative to the low spatial frequency stimuli) increased neural activity in the primary visual cortex bilaterally (local maximum: $x=4, y=-102, z=0 ; z$-score $=4.0, P<0.001)$. No significant increase in cerebral activity in any area was observed with low spatial frequency stimuli (when compared to relatively high spatial frequency stimuli).

The main effects of processing level (globally vs locally directed attention and vice-versa) showed no significant increase in neural activity either during global or during local processing in early visual areas. An unpredicted but significant $(P<0.05$, corrected) activation was observed in the left superior posterior parieto-occipital cortex during local processing (relative to global processing). The local maximum of this region was: $x=-22, y=-74$, $z=40 ; z$-score $=4.6$. The region corresponds to Brodmann Area 19

In the absence of significant activations in early visual areas due to processing level, we next assessed the interaction between processing level and stimulus level. This interaction term is the difference between globally and locally directed attention in relatively high and relatively low spatial frequency stimuli and vice-versa (i.e. high spatial frequency stimuli globally directed attention (HG) - high spatial frequency, locally directed attention $(\mathrm{HL})>$ low spatial frequency, globally directed attention (LG) - low spatial frequency, locally directed attention (LL), and vice-versa). Significant interactions were observed in the left inferior occipital cortex abutting on the fusiform gyrus (local maximum: $x=-32, y=-82$, $z=-12 ; z$-score $=3.9, P<0.001 ;$ Fig. $2 \mathrm{a})$ and the right lingual gyrus (local maximum: $x=16, y=70, z=-4$; $z$-score $=3.9, P<0.001 ;$ Fig. 2 a) within the predicted early visual processing areas implicated in global/local processing [5]. The nature of this interaction explains why no main effect of global/local processing across both stimulus types (high and low spatial frequency) was found in these areas. The pattern of activation can be clearly seen when the simple effects of global/local processing in both relatively high and relatively low spatial frequency conditions are calculated separately (Fig. 2b and 2c).

Relative rCBF increases associated with the globally directed attention task with relatively high spatial frequency stimuli $(\mathrm{HG}>\mathrm{HL})$ were seen in the right lingual gyrus (local maximum: $x=18, y=-62, z=-8 ; z$-score $=3.7$, $P<0.001$; Fig. 2b). Locally directed attention with relatively low spatial frequency stimuli $(L L>L G)$ activated the left inferior occipital cortex alone (local maximum: $x=-28, y=-84, z=-16 ; z$-score $=4.4, P<0.001$; Fig. 2c). Relative activations in the respective homologous contralateral regions did not reach significance. To assess the observed hemispheric asymmetries in $\mathrm{rCBF}$ responses, hemisphere $\times$ condition interactions were examined in the above regions that were identified on the basis of the (independent) simple effects of each condition. The direct comparison of $\mathrm{rCBF}$ changes between the two hemispheres indicated that both lateralization effects were statistically significant $(P<0.005)$.

The regions activated in the present study are strikingly 


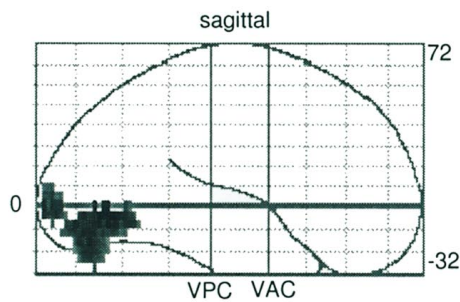

$-104$

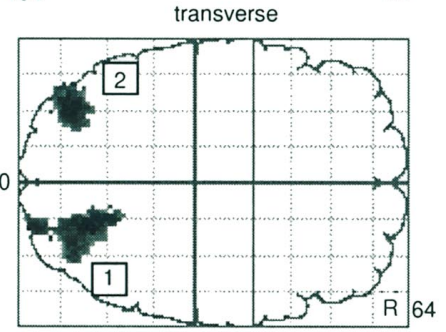

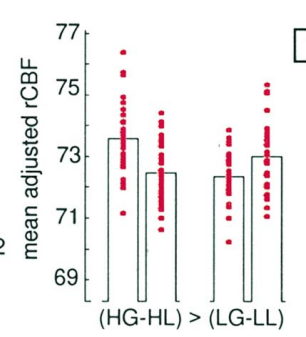
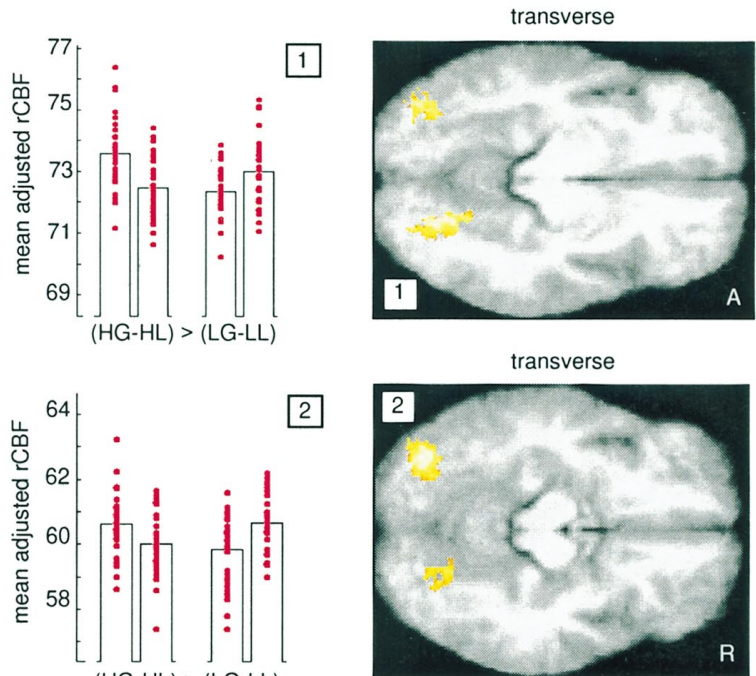

2

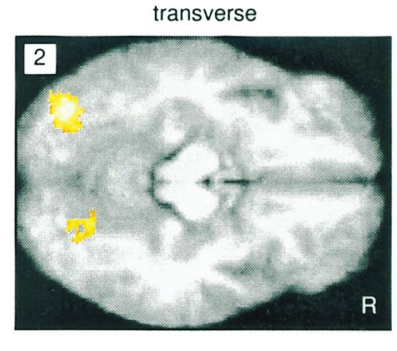

interaction of high / low spatial frequency stimuli on globally and locally directed attention
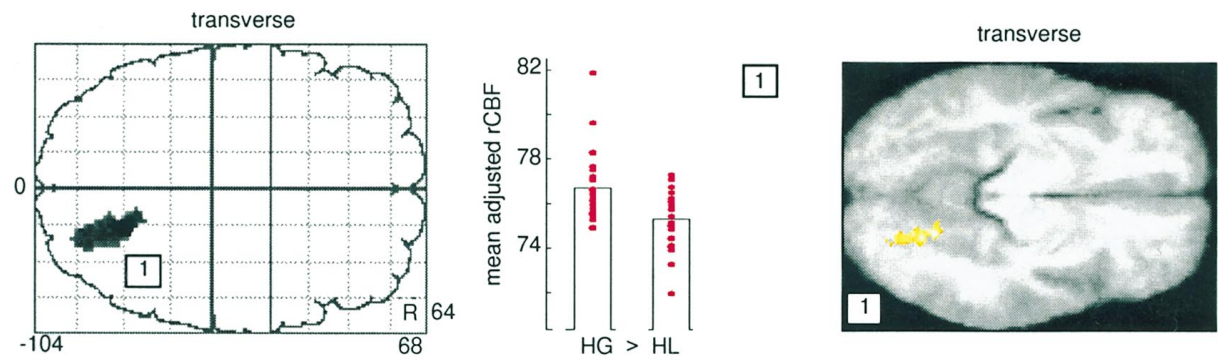

b

high spatial frequency stimuli: globally directed attention
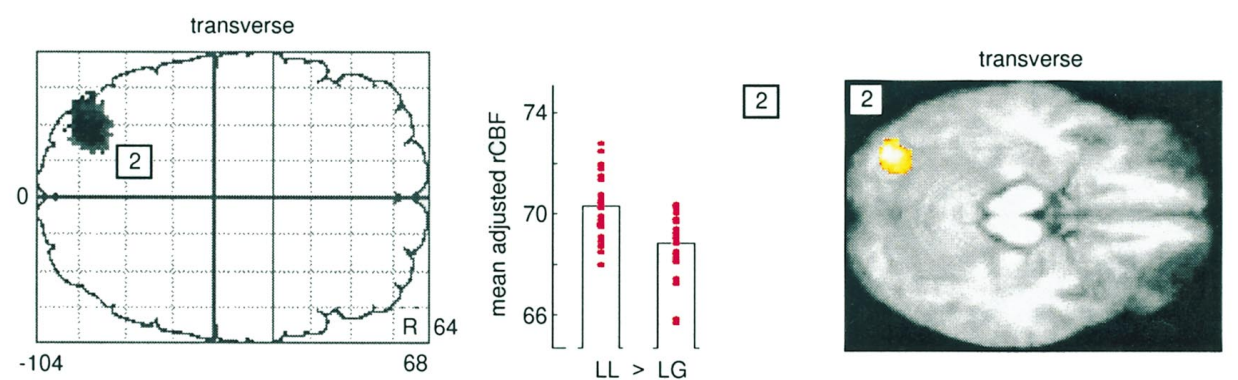

low spatial frequency stimuli: locally directed attention

C

Fig. 2. Relative activations for the 10 subjects associated with (a) the interaction of high/low spatial frequency stimuli on globally and locally directed attention [(HG-HL) > (LG-LL)], (b) globally directed attention with high spatial frequency stimuli (HG > HL) and (c) locally directed attention with low spatial frequency stimuli ( $\mathrm{LL}>\mathrm{LG}$ ). Areas of significant activations are shown as through-projections onto representations of standard stereotactic space. Sagittal, side view; transverse, view from above. To detail the functional anatomy of the activations and their relationship to structural anatomy, the respective $\mathrm{SPM}_{\{z\}}$ maps were superimposed upon the group mean MR-image (transverse sections taken through the respective local maximum). The exact coordinates of the local maxima (indicated by the number in each box) within the areas of activation and their $z$ statistics are given in the text. In addition, adjusted mean $\mathrm{rCBF}$ and the individual $\mathrm{rCBF}$ values per condition are displayed for the respective pixel of maximally significant relative $\mathrm{rCBF}$ increase within the respective areas of interest. $\mathrm{R}=$ right, $\mathrm{A}=$ anterior, VAC $=$ vertical plane through the anterior commissure, numbers at axes refer to coordinates of standard stereotactic space, $\mathrm{HG}=$ high spatial frequency stimuli/globally directed attention, $\mathrm{HL}=$ high frequency stimuli/locally directed attention, $\mathrm{LG}=$ low frequency stimuli/globally directed attention, $\mathrm{LL}=$ low frequency stimuli locally directed attention. 
similar to those previously identified in a similar designed functional imaging study using letter-based hierarchical stimuli $[5,6]$. These areas lie in the extrastriate cortex and correspond to more peripheral (right lingual gyrus) and more foveal (left inferior occipital cortex) parts of areas V2/V3 [34, 36].

\subsection{Discussion}

Differential spatial frequency tuning of the cerebral hemispheres has been conjectured to play a major role in the explanation of hemispheric asymmetries in processing global or local aspects of visual stimuli [13]. Sergent [35] suggested that, all else being equal a cognitive task that stressed high spatial frequency information would result in a right visual field/left hemisphere advantage and a task that stressed low spatial frequency information would result in a left visual field/right hemisphere advantage. Shulman et al. [37] have subsequently claimed that differences in tuning to low- and high-spatial frequencies ultimately determine hemispheric specificity in global and local processing. Our results constrain the generality of this hypothesis $[15,37]$ by demonstrating that global processing of relatively high spatial frequency stimuli leads to augmented activation in the right lingual gyrus and that local processing of relatively low spatial frequency stimuli leads to augmented activation in the left interior occipital cortex. Thus the simple notion of a left hemispheric advantage for relatively high spatial frequency/local processing and a right hemisphere advantage for relatively low spatial frequency/global processing is not confirmed by our neurophysiological measurements. Although our relatively high spatial frequency stimuli are within the low absolute range, there is a consensus that the left and right hemispheres are specialized for the processing of relatively high and low frequencies respectively and not necessarily for high and low frequencies in some absolute sense $[13,30]$. Our findings, however, support other recent evidence that mechanisms regulating the perception of local and global properties and of high and low spatial frequencies, although often intercorrelated, are not equivalent [10].

Inspection of our stimulus material (Fig. 1) suggests that the global form (the overall Gestalt) is more prominent than the local components in the high spatial frequency stimuli. The small (white) "gaps" do not seem to perturb the percept of one (horizontal or vertical) rectangle. By contrast, the (three) local forms appear more prominent than the global form in the low spatial frequency stimuli. The larger white "gaps" (relative to those in the high spatial frequency stimuli) break up the Gestalt into its separate local components.

If the above conjectures are valid, our results suggest that early visual areas in the left hemisphere are specialized for local processing, while early visual areas in the right hemisphere are specialized for global processing, if the respective features of non-representational stimuli are perceptually salient (i.e. perception of the relevant feature, local or global is facilitated by the structure of the stimulus). However, if this alternative conclusion is correct, then it predicts that the relatively high spatial frequency stimuli used in experiment 1 are globally more salient and that the relatively low spatial frequency stimuli are locally more salient. Accordingly, we tested this prediction by designing an independent reaction time study (performed outside the scanning environment) to investigate directly the relative salience of the two types of stimuli with respect to the two different tasks.

\section{Experiment 2: a reaction time study}

\subsection{Introduction}

If our conjecture is correct, the proposed differences in perceptual salience of the global or local attributes of the stimuli used in experiment 1 predict faster reaction times to the more salient level (global or local). Specifically, one would predict that the more salient local level in the low spatial frequency stimuli will be easier to report (and hence quicker to react to) than the less salient global level of the low spatial frequency stimuli. Accordingly, the well-known finding that the global level is usually processed quicker in Navon letters than the local level (global precedence $[12,15]$ ) would be reversed. Global precedence, however, should be preserved in the relatively high spatial frequency stimuli where the global attribute is conjectured to be more salient than the local attribute.

\subsection{Methods}

\subsubsection{Subjects}

Twelve normal-sighted healthy right-handed volunteers (11 male, 1 female; aged 23-33 years) were recruited.

\subsubsection{Paradigm design}

The experimental design and stimuli used were identical to experiment 1 (Fig. 1). There were 4 conditions, each with 3 repeats, per subject. Accordingly, there were 144 blocks of trials (4 conditions $\times 3$ repeats $\times 12$ subjects). The only methodological difference was that in experiment 2 subjects were asked to press a response key with either their left or right index finger in order to indicate the orientation (horizontal or vertical) of the processing level (global or local) demanded in a particular block of trials. Half the subjects indicated "horizontal" with the left index finger and "vertical" with the right index finger. These responses were reversed for the other six subjects. 


\subsubsection{Procedure}

Subjects were seated in front of a computer monitor $\left(17^{\prime \prime}\right.$ VDU) at a viewing distance of $40 \mathrm{~cm}$. Immediately in front of each subject there was a custom-made response key by the left and right hand of the subject. The experiment was run on an Apple Macintosh Power PC and controlled by the same software used for the PET experiment and modified to record reaction times. Reaction time (RT) was measured from stimulus onset to the pressing of a response key. As in the PET experiment (experiment 1), prior to the RT experiment all subjects were made fully familiar with the stimuli and tasks in a series of practice trials that continued until the subject was satisfied that she/he understood what was required.

\subsection{Results}

The mean number of errors per subject (per condition per block of 80 trials) was low: high spatial frequency global 1.8 (mean) \pm 1.8 (sdev), high spatial frequency local $1.8 \pm 1.6$, low spatial frequency global $1.8 \pm 1.7$ and low spatial frequency local $2.2 \pm 1.6$. These differences in error rate between the four conditions were not statistically significant.

Mean reaction times and standard deviations for the four conditions were: high spatial frequency global 524 ms (mean) $\pm 92 \mathrm{~ms}$ (sdev), high spatial frequency local $612 \mathrm{~ms} \pm 61 \mathrm{~ms}$, low spatial frequency global $569 \mathrm{~ms} \pm 48$ $\mathrm{ms}$ and low spatial frequency local $576 \mathrm{~ms} \pm 49 \mathrm{~ms}$. On ANOVA ( 4 conditions $\times 3$ repeats $\times 12$ subjects) there was no significant main effect of spatial frequency. By contrast, processing level was significant as a main effect $(F(1,140)=19.1, P<0.0001)$. Finally, there was a significant interaction of spatial frequency and processing level $(F(1,140)=13.9, P<0.0005)$. The nature of this interaction is shown graphically in Fig. 3.

\subsection{Discussion}

When subjects performed the global task, responses to high frequency stimuli were significantly faster than to low spatial frequency stimuli. By contrast, in the local task, responses to low spatial frequency stimuli were made significantly faster than to high spatial frequency stimuli. The form of this interaction is equivalent to that found in the PET study (experiment 1) and provides independent evidence that the primary determinant of performance within each task is visual salience. The effect of high spatial frequency is to render the global form more salient: decreasing the distance between the component rectangles of the stimulus strengthens the boundaries of the figure as a whole [14]. Conversely, the effect of low spatial frequency is to render the local forms more salient: increasing the distance between the component rectangles renders each individual rectangle more prominent.

\section{General discussion}

Experiment 1 (the PET study) was not designed to evaluate the role of temporo-parietal cortex in global/local processing [22, 32]. This issue has previously been dealt with in both lesion $[32,33]$ and functional imaging studies [5, 6]. Rather, experiment 1 supports previous evidence from both PET and fMRI that global/local processing of many different stimulus types (letters, objects, and patterns) is also associated with differential hemispheric activation of early visual areas [5-7, 11, 24]. In previous articles we have argued that these early visual processing mechanisms in prestriate cortex are influenced by an attentional system of the type conjectured by Robertson and Lamb [32] and mediated by temporo-parietal areas [5-7].

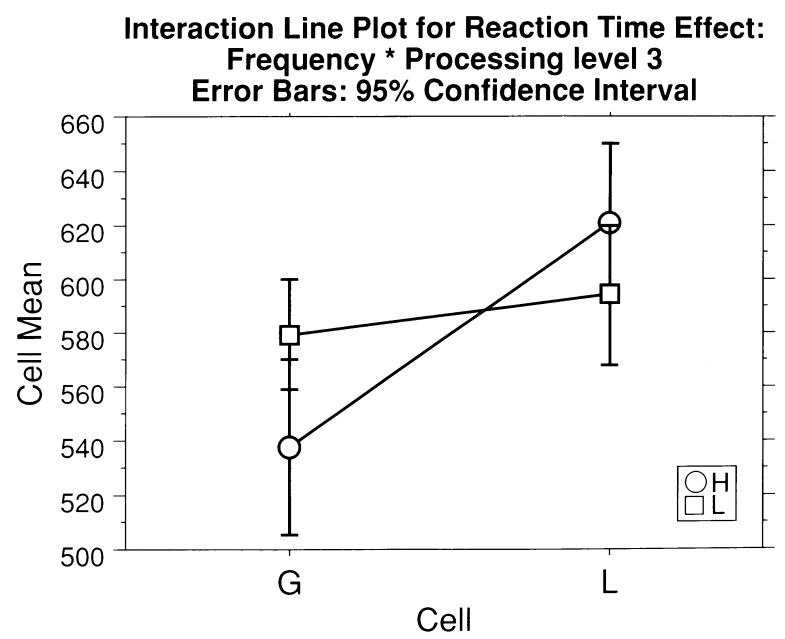

Fig. 3. Mean reaction times and 95\% confidence intervals for the four conditions: high spatial frequency global, high spatial frequency local, low spatial frequency global and low spatial frequency local. On ANOVA there was no significant main effect of spatial frequency. By contrast, processing level was significant as a main effect. There was also a significant interaction of spatial frequency and processing level. 
The current results, however, argue against the claim that local and global visual processing per se are invariably associated with left and right hemisphere activation, respectively. Rather, the observed differential activations in early visual processing areas support other claims that the hemisphere maximally involved in a particular test is determined by interactions of processing level and stimulus characteristics $[10,19,30]$. There is, however, one region that we found to be significantly activated as a main effect of local processing. This region (BA 19) is known to be visual association cortex [43]. We suggest that its activation in our study may reflect the additional perceptual/attentional processing required to overcome the global precedence effect when the task demands analysis of local features of the non-representational spatial frequency stimuli used in this experiment.

The stimulus characteristic that plays the major role in experiment 1 appears to be perceptual salience. This feature is most easily quantified by taking reaction times for performing the global/local tasks with the high/low spatial frequency stimuli (experiment 2). Experiment 2 confirms that the saliency interpretation of the results of experiment 1 is highly plausible. The psychophysical data and functional imaging data thus provide compelling mutual support. Visual salience (ease of perception) is clearly influenced in our experiment by relative spacing. There are no doubt many other factors concerning Gestalt effects of good form [20] that can also determine salience. The scope of the current data is limited to the manipulation of relative spacing/spatial frequency.

The stimuli we presented were invariant across the relevant comparisons, with the only changes being the instructions to subjects. Our data accordingly add to existing evidence for the hypothesis that neural activity in early visual areas does not solely represent the physical attributes of a retinal image but is also influenced by attentional modulation via "top-down" (endogenous) processes [3, 26, 27, 38]. In our study, changes in spatial frequency, which determine perceptual salience for the two tasks, interact with neural activations due to following instructions to report either global or local stimulus properties. Thus, bottom-up (exogenous) and topdown (endogenous) processes interact in extrastriate cortical regions to construct a coherent percept. Brown and Kosslyn [1] have argued in relation to hemispheric specialization that "rather than expecting dichotomies that reflect general processing differences (such as analytic vs holistic), the literature suggests that there will be more specific principles that extend only over limited domains". Our results are consistent with their position: Non-representational local and global forms are processed preferentially by early visual areas of the left and right hemisphere respectively, under the constraint that the relevant visual property is salient. It remains the case, however, that elucidation of the precise mechanisms involved may require greater attention to the particular psychophysical properties of the stimulus set that determine salience. Our stimuli are truncated (co)sine gratings on one axis and large spectra patterns on the other axis (i.e. the ends of each component line were not graded). We chose these stimuli because of their similarity to stimuli previously used in psychophysical experiments comparable to our own [17], but in future work it may be necessary to deploy, for example, Gabor patches that model the known receptive-field properties of neurons in primary visual cortex.

Finally, we note that in future experimentation (and theory) concerning global/local processing it will be necessary to investigate stimuli that are more naturalistic than those introduced by Navon [28] and deployed by so many subsequent workers. When describing informally what one means by levels of global/local processing it is common to discuss such examples as a forest constituted by trees which in turn have branches which in turn have leaves, or a bridge made of bricks [5, 18]. Hierarchical levels of this nature are not necessarily equivalent to large letters made of small letters or, as in experiment 1, a large rectangle made of small rectangles. Likewise the global and local features of a face are different from global and local levels of a hierarchical Navon figure. Similarly, the distinction that Kimchi [18] draws between "global properties, defined by their position in the hierarchical structure of the stimulus, and wholistic properties defined as a function of interrelations among component parts" may prove crucial in future work. What is salient in a hierarchical Navon figure may be different from what is salient in stimuli with a more realistic appearance.

\section{Acknowledgements}

We thank our volunteers and the radiography staff at the Wellcome Department. We are grateful to J. Heather for the stimulus software and to W. J. M. Levelt for the idea that prompted this study. P. W. H. and J. C. M. are supported by the Medical Research Council. R. J. D. and G. R. F. are supported by the Wellcome Trust.

\section{References}

[1] Brown BG, Kosslyn SM. Cerebral lateralisation. Current Opinion in Neurobiology 1993;3:183-186.

[2] Corbetta M, Miezin FM, Dobmeyer S, Shulman GL, Petersen SE. Selective and divided attention during visual discriminations of shape, color, speed functional anatomy by positron emission tomography. Journal of Neuroscience 1991;11:2383-2402.

[3] Dolan RJ, Fink GR, Rolls E, Booth M, Holmes A, Frackowiak RSJ, Friston KJ. How the brain learns to see objects and faces in an impoverished context. Nature 1997;389:596-599.

[4] Fink GR, Dolan PJ, Halligan PW, Marshall JC, Frith CD. Spacebased and object-based attention: Shared and specific neural domains. Brain 1997;120:2013-2028,

[5] Fink GR, Halligan PW, Marshall JC, Frith CD, Frackowiak RSJ, 
Dolan RJ. Where in the brain does visual attention select the forest and the trees? Nature 1996;382:626-628.

[6] Fink GR, Halligan PW, Marshall JC, Frith CD, Frackowiak RSJ, Dolan RJ. Neural mechanisms involved in the processing of global and local aspects of hierarchically organized visual stimuli. Brain 1997;120:1779-1791.

[7] Fink GR, Marshall JC, Halligan PW, Frith CD, Frackowiak RSJ, Dolan RJ. Hemispheric specialization for global and local processing: the effect of stimulus category. Proceedings of the Royal Society London B 1997;264:487-494.

[8] Friston KJ, Ashburner J, Frith CD, Poline J-B, Heather JD, Frackowiak RSJ. Spatial registration and normalization of images. Human Brain Mapping 1995;2:1-25.

[9] Friston KJ, Holmes AP, Worsley K J, Poline, J-B, Frith CD, Frackowiak RSJ. Statistical parametric maps in functional imaging: a general linear approach. Human Brain Mapping 1995;2:189 210 .

[10] Grabowska A, Nowicka A. Visual-spatial-frequency model of cerebral asymmetry: a critical survey of behavioral and electrophysiological studies. Psychological Bulletin 1996;120:434-449.

[11] Heinze HJ, Münte TF. Electrophysiological correlates of hierarchical stimulus processing: dissociation between onset and later stages of global and local target processing. Neuropsychologia 1993;31:841-852

[12] Hellige, JB. Hemispheric Asymmetry: What's Right and What's Left. Harvard University Press, Cambridge, Massachusetts 1993.

[13] Hellige JB. Hemispheric asymmetry for components of visual information processing. In Davidson RJ, Hughdahl $\mathrm{K}$ editor. Brain Asymmetry,Massachusetts Institute of Technology, Massachusetts 1995, pp. 99-122.

[14] Hoffman DD, Singh M. Salience of visual parts. Cognition 1997;63:29-78.

[15] Hughes HC, Nozawa G, Kitterle F, Global precedence, spatial frequency channels, the statistics of natural images. Journal of Cognitive Neuroscience 1996;8:197-230.

[16] Hurlbert A, Poggio T. Spotlight on attention. Trends in Neuroscience 1985;63:309-311.

[17] Ivry RB, Robertson LC. The two sides of perception. MIT Press, Cambridge, MA 1998.

[18] Kimchi R. Primacy of wholistic processing and global/local paradigm: A critical review. Psychological Bulletin 1992;112:24-38.

[19] Kinchla RA, Solis-Macias V, Hoffman J. Attending to different levels of structure in a visual image. Perception and Psychophysics 1983:33:1-10.

[20] LaGasse LL. Effects of good form and spatial frequency on global precedence. Perception and Psychophysics 1993;53:89-105.

[21] Lamb MR, Robertson LC. The processing of hierarchical stimuli: Effects of retinal locus, locational uncertainty and stimulus identity. Perception and Psychophysics 1988;44:172-181.

[22] Lamb MR, Robertson LC, Knight RT. Component mechanisms underlying the processing of hierarchically organized patterns: Inferences from patients with unilateral cortical lesions. Journal of Experimental Psycholology (Learning, Memory, Cognition) 1990; $16: 471-483$.

[23] Lavie N. Perceptual load as a necessary condition for selective attention. Journal of Experimental Psychology: Human Perception and performance 1995;21:451-468.

[24] Martinez A, Moses P, Frank L, Buxton R, Wong E, Stiles J. Hemispheric asymmetries in global and local processing: evidence from fMRI. Neuroreport 1997;8:1685-1689.
[25] Mazziotta JC, Huang SC, Phelps ME, Carson RE, MacDonald NS, Mahoney, K. A non-invasive positron computed tomography technique using oxygen-15 labelled water for the evaluation of a neurobehavioral task battery. Journal of Cerebral Blood Flow and Metabolism 1985;5:70-78.

[26] Moran J, Desimone R. Selective attention gates visual processing in the extrastriate cortex. Science 1985;229:782-784.

[27] Motter BC. Focal attention produces spatially selective processing in visual cortical areas V1 V2, V4 in the presence of competing stimuli. Journal of Neurophysiology 1993;70:909-919.

[28] Navon D. Forest before trees: The precedence of global features in visual perception. Cognitive Psychology 1977;9:353-383.

[29] Polster MR, Rapcsak SZ. Hierarchical stimuli and hemispheric specialization: two case studies. Cortex 1994;30:487-497.

[30] Rafal R, Robertson LC. The neurology of visual attention. In Gazzaniga MS, editor. The Cognitive Neurosciences, MIT Press, London 1995, pp. 625-648.

[31] Raichle ME. Circulatory and metabolic correlates of brain function in normal humans. In Handbook of Physiology. Vol. 5. The Nervous System. Part 1. Higher Functions of the Brain, ed. V. B. Mountcastle. American Physiological Society, Bethesda 1987, pp. 643-674.

[32] Robertson LC, Lamb MR. Neuropsychological contributions to theories of part/whole organization. Cognitive Psychology 1991;23:299-330.

[33] Robertson LC, Lamb MR, Knight RT. Effects of lesions of temporal-parietal junction on perceptual and attentional processing in humans. Journal of Neuroscience 1988;8:3757-3769.

[34] Sereno MI, Dale AM, Reppas JB, Kwong KK, Belliveau JW, Brady TJ, Rosen B. R, Tootelt RBH. Borders of multiple visual areas in humans revealed by functional magnetic resonance imaging. Science 1995;268:889-893.

[35] Sergent J. Role of the input in visual hemispheric asymmetries. Psychological Bulletin 1983;93:481-512.

[36] Shipp S, Watson JDG, Frackowiak, RSJ, Zeki S. Retinotopic maps in human prestriate visual cortex: the demarcation of areas V2 and V3. Neuroimage 1995;2:125-132.

[37] Shulman G, Sulivan M, Gish K, Sakoda W. The role of spatial frequency channels in the perception of local and global structure. Perception 1986;15:259-273.

[38] Shulman GL, Corbetta M, Buckner RL, Raichle ME, Fiez JA, Miezin FM, Petersen SE. Top-down modulation of early sensory cortex. Cerebral Cortex 1997;7:193-206.

[39] Silbersweig DA, Stern E, Frith CD, Cahill C, Schnorr L, Grootonk S, Spinks T, Clark J, Frackowiak, RSJ, Jones T. Detection of thirty-second cognitive activations in single subjects with positronemission-tomography: a new lowdose $\mathrm{H}_{2}{ }^{15} \mathrm{O}$ regional cerebral blood flow three-dimensional imaging technique. Journal of Cerebral Blood Flow and Metabolism 1993;13:617-629.

[40] Spitzer H, Desimone R, Moran J. Increased attention enhances both behavioral and neuronal performance. Science 1988;240:338 340 .

[41] Talairach J, Tournoux P. Coplanar Stereotaxic Atlas of the Human Brain. Thieme, Stuttgart 1988.

[42] Townsend DW, Geissbuller A, Defrise M, Hoffman EJ, Spinks TJ, Bailey DL, Gilarm di M-C, Jones T. Fully three-dimensional reconstruction for a PET camera with retractable septa. IEEE Transactions on Medical Imaging 1991;10:505-512.

[43] Zeki S. A Vision of the Brain. Blackwell Scientific Publication, Oxford 1993. 